Mini Review: Open Access

\title{
The Diagnostic Role of Cardiac Magnetic Resonance Imaging in Rheumatoid Arthritis
}

\author{
H Kobayashi ${ }^{1 *}$, Y Kobayashi ${ }^{2}$, S Ode ${ }^{3}$, K Sugiyama ${ }^{1}$, K Kitamura $^{1}$ and M Takei' \\ ${ }^{1}$ Department of Medicine, Division of Hematology and Rheumatology, Nihon University School of Medicine, Japan \\ ${ }^{2}$ Department of Advanced Biomedical Imaging Informatics, St. Marianna University School of Medicine, Kawasaki, Japan \\ ${ }^{3}$ Department of Radiology, St.Marianna University School of Medicine, Kawasaki, Japan
}

${ }^{*}$ Corresponding author: H. Kobayashi, MD, PhD Department of Medicine, Division of Hematology and Rheumatology, Nihon University School of Medicine, 30-1 Oyaguchi Itabashi-Ward, Tokyo 173-8610, Japan, Tel: 8133972 8111, Fax: 8133972 2893, E-mail: kobayashi.hitomi48@nihon-u.ac.jp

\begin{abstract}
In patients with rheumatoid arthritis (RA), cardiac involvement such as myocarditis and myocardial infarction is common. This cardiac involvement may have serious consequences and can contribute to worsening of a patient's cardiac-related morbidity and mortality. Importantly, cardiac involvement is typically clinically silent, only manifesting as cardiac dysfunction after an extended preclinical phase. Cardiovascular magnetic resonance (CMR) is a sensitive noninvasive diagnostic technique that can identify subclinical cardiac structural and functional abnormalities. MRI can be performed with repeatedly for diagnosis and follow-up in collagenvascular diseases, which mostly affect relatively young individuals. We present a short review of the diagnostic role of CMR in patients with RA.
\end{abstract}

\section{Keywords}

Rheumatoid arthritis, Cardiac magnetic resonance imaging, Cardiac involvement

\section{Introduction}

Rheumatoid arthritis (RA) is a multi-organ disease that presents with not only articular symptoms but also various other clinical manifestations. In Western countries, life expectancy is approximately 10 years shorter in patients with RA than in healthy individuals, and cardiac involvement is the leading cause of death in those with RA [13]. However, even in the presence of cardiac involvement, RA is more likely to be asymptomatic, and compromises the vital prognosis [4]. Recently, cardiac involvement has also become a major cause of death in patients with RA in Japan [5].

With advances in cardiac magnetic resonance imaging (CMR), the assessment of regional or global cardiac function (contractility and diastolic function), myocardial ischemia, the condition of the myocardium, the coronary arteries and before myocardial ischemia can be performed in clinical settings; the detection of cardiac lesions can be performed with noninvasive manner, and the severity of the lesions can be quantified [6]. Furthermore, CMR provides better detection capability than nuclear testing for small myocardial infarctions and myocardial ischemia due to lesions in the three main coronary artery branches
[7]. Furthermore, contrast-enhanced CMR is recognized as a valuable tool for the diagnosis of myocardial diseases. Unlike nuclear tests and computed tomography (CT), MRI causes no radiation exposure; therefore, MRI can be performed with repeatedly for diagnosis and follow-up in collagen-vascular diseases, which mostly affect relatively young individuals. Compared to echocardiography, this test has high reproducibility, with stable measurements $[8,9]$. It is particularly useful for measuring left ventricular (LV) mass in CMR. Because the entire LV volume is evaluated by measuring the LV mass and the volume of the cardiac chamber in CMR, CMR might be a better option to precisely measure the LV geometry $[10,11]$.

Its detection and histological confirmation are often difficult because of a lack of symptoms in RA. CMR might be considered a useful and less invasive diagnostic method for assessing cardiac involvement in asymptomatic patients with RA. This paper provides a short review focused on the role of CMR in RA patients.

\section{The Basics of Cardiac MRI}

Alone among diagnostic imaging modalities, MRI allows not only morphological evaluation, but also enables simultaneous functional diagnosis and noninvasive assessment of myocardial properties. CMR has several positive features. (1) The test is minimally invasive and causes no radiation exposure. (2) In addition to allowing cardiac morphological diagnosis, the test also uses various sequences and enables functional diagnosis of cardiac motion and myocardial ischemia. (3) Allergic reactions to gadolinium-based MRI contrast agents are believed to be less common than those due to iodinated contrast agents used in CT and angiography [12]. However, CMR also has problematic features. (1) The tests are time-consuming. (2) Artifacts often occur when the tests are conducted on arrhythmia patients. (3) Experience is needed to interpret the results. (4) A contrast agent is often needed, and patients with allergies or impaired renal function are at risk of developing nephrogenic systemic fibrosis [13].

CMR utilizes various sequences as described below, and the choice of sequence depends on the purpose.

\section{Cine MRI and tagging MRI}

Global function: This enables evaluation of ejection fraction, end-

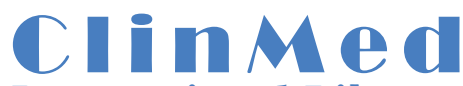

International Library

Citation: Kobayashi H, Kobayashi Y, Ode S, Sugiyama K, Kitamura N, et al. (2016) The Diagnostic Role of Cardiac Magnetic Resonance Imaging in Rheumatoid Arthritis. Int J Immunol Immunother 3:019

Received: June 19, 2016: Accepted: June 28, 2016: Published: June 30, 2016

Copyright: (c) 2016 Kobayashi $\mathrm{H}$, et al. This is an open-access article distributed under the terms of the Creative Commons Attribution License, which permits unrestricted use, distribution, and reproduction in any medium, provided the original author and source are credited. 
diastolic volume, and end-systolic volume, as well as measurement of myocardial mass

Regional function: This enables quantitative evaluation as well as visual assessment. Tagging MRI or feature tracking MRI, permit measurement of the local strain value of myocardium. With recent software development, feature tracking analysis also enables strain measurement using cine MRI.

Black-blood T2-weighted imaging (BB T2-WI): This visualizes myocardial edema as a high-signal area, and can assess myocarditis and acute myocardial infarction. BB T2-WI shows active inflammation in the myocardium.

Late gadolinium enhancement (LGE): In this phenomenon, the contrast effect can be observed in the myocardium approximately 10 15 minutes after intravenous injection of gadolinium-based contrast agent. In the early stages after administration, the contrast agent is distributed in the myocardial vascular bed, but later leaks into the interstitium and fills the extracellular space, results in delayed washout. The LGE indicates myocardial fibrosis, myocardial infarction and severe myocarditis.

ECV and T1 mapping: With recent advance of cardiac MRI, myocardial inflammation and diffuse fibrosis can be detected noninvasively by using native T1 mapping and extracellular volume (ECV) quantification on CMR.

\section{Proper Combination of Sequences in RA}

If the findings show pathological features (myocarditis and fibrosis) that are assumed to be associated with cardiac lesions in RA patients, further evaluation can include a combination of cine MRI + BB T2-WI + LGE (if possible, + T1 mapping) when there are no clear symptoms of ischemia; with this combination, CMR can be included among routine laboratory tests.

\section{Research Study on CMR in RA}

Until now, CMR has been successfully used for the evaluation of myocardial inflammation in different types of vasculitis $[14,15]$, myositis [16], and SLE [17,18]. Giles et al. demonstrated the evaluation of left ventricular structure and function in patients with RA, as assessed by CMR [19]. They suggest that the progression to heart failure in RA may occur through reduced myocardial mass rather than hypertrophy.

We demonstrated that CMR was performed in RA patients without known heart disease, and the findings showed potential cardiac lesions in a pilot study [20]. LGE was observed in seven patients (38.9\%). Perfusion defects under pharmacologic stress were observed in two patients. Mean DAS28 was significantly higher in the group with LGE compared to the group without by an average of 1.32 DAS28 units. Myocardial abnormalities, as detected by CMR were frequent in RA patients without known cardiac disease. Abnormal CMR findings were associated with higher RA disease activity, suggesting a role for inflammation in the pathogenesis of myocardial involvement in RA. Furthermore, we also demonstrated that regional function in RA patients was apparently lower than that found in healthy subjects assessed using CMR [21]. In addition, global cardiac function and left ventricular mass (LVM) and geometry were examined by using CMR in healthy subjects and RA patients without cardiac symptoms, matched according to age and sex; in comparison with healthy subjects, RA patients had a lower ejection fraction, increase LVM, and altered geometry [22]. When the patients were administered the biological product tocilizumab for one year, cardiac function improved, and LVM and geometry also showed improvement. Multivariate analyses revealed that disease activity in RA contributed the most to the improvements. Our study also showed improvement in regional function, associated with disease activity in RA, after a biological product was administered for one year [21]. This suggested that cardiac function and geometry associated with RA might be caused by RA itself (through inflammatory pathways mediated by cytokines). The findings also suggested that when RA was in the highly active phase, the resulting cardiac involvement remained subclinical, but had an impact on cardiac function, and could be diagnosed by CMR.

However, LGE relies on the comparison of focal myocardial damage against unaffected normal myocardium. Therefore, it is difficult to detect diffuse myocardial fibrosis. ECV and T1 mapping has proved to be a robust tool for the noninvasive assessment of diffuse myocardial fibrosis. Ntsui et al. reports that patients with RA had larger areas of focal myocardial edema, higher native T1 values and expansion of ECV compared with control subjects, and myocardial T1 and ECV were correlated with myocardial strain and RA disease activity [23]. Recently, Holmström $\mathrm{M}$ et al. demonstrated that myocardial $\mathrm{T} 1$ relaxation times are prolonged suggesting diffuse inflammation or fibrosis in RA and local myocardial scars and inflammation, visible as LGE, are also common, as are impairments of LV systo-diastolic function [24]. Miszalski-Jamka T et al. showed that cardiac involvement are common in Churg-Strauss syndrome and granulomatosis with polyangiitis (Wegener's) patients with normal electrocardiograms and transthoracic echocardiography, evaluating by using standard and feature tracking magnetic resonance [25]. Further studies of this diagnostic tool should be evaluated to discuss its utility.

\section{Clinical Use of CMR in CVD due to RA}

In general, cardiac involvement due to RA has features that are different from cardiac involvement caused by other diseases. RA patients reportedly have fewer typical cardiac symptoms. Kremers et al. showed that RA patients have a significantly higher risk of cardiac involvement when compared with non-RA subjects. They also indicated that RA patients are less likely to report symptoms of cardiac involvement and sudden cardiac death [4]. This evidence showed the importance of early assessment for subclinical heart disease in RA patients. Research studies on the stratification of highrisk patients with subclinical heart disease will be needed even more in the future; if heart disease could be diagnosed at an early stage with CMR, the prevention and treatment of CVD associated with RA would be followed. Rheumatologists should always consider the possibility of CVD as a complication of RA, in order to advance the diagnosis of subclinical heart disease.

*All authors attest that they have no financial conflicts of interest pertaining to this investigation

\section{References}

1. Wolfe F, Mitchell DM, Sibley JT, Fries JF, Bloch DA, et al. (1994) The mortality of rheumatoid arthritis. Arthritis Rheum 37: 481-494.

2. Doran MF, Pond GR, Crowson CS, O'Fallon WM, Gabriel SE (2002) Trends in incidence and mortality in rheumatoid arthritis in Rochester, Minnesota, over a forty-year period. Arthritis Rheum 46: 625-631.

3. Douglas KMJ, Pace AV, Treharne G J, Saratzis A, Nightingale P, et al. (2006) Excess recurrent cardia events in rheumatoid arthritis patients with acute coronary syndrome. Ann Rheum Dis 65: 348-353.

4. Kremers MH, Crowson CS, Nicola PJ, Ballman KJ, Roger VL, et al. (2005) Increased unrecognized coronary heart disease and sudden deaths in rheumatoid arthritis: a population-based cohort study. Arthritis Rheum 52: 402-411.

5. Nakajima A, Inoue E, Tanaka E, Singh G, Sato E, et al. (2010) Mortality and cause of death in Japanese patients with rheumatoid arthritis based on a large observational cohort, IORRA. Scand J Rheumatol 39: 360-367.

6. Tzelepis GE, Kelekis NL, Plastiras SC, Mitseas P, Economopoulos N, et al. (2007) Pattern and distribution of myocardial fibrosis in systemic sclerosis: a delayed enhanced magnetic resonance imaging study. Arthritis Rheum 56: 3827-3836.

7. Greenwood JP, Motwani M, Maredia N, Brown JM, Everett CC, et al. (2012) Cardiovascular magnetic resonance and single-photon mission computed tomography for diagnosis of coronary heart disease(CE-MARC):a prospective trial. Lancet 379: 453-460.

8. Maksimovic R, Seferovic PM, Ristic AD, Vujisic-Tesic B, Simeunovic DS, et al. (2006) Cardiac imaging in rheumatic diseases. Rheumatology 45: iv26-31.

9. Mavrogeni S, Vassilopoulos D (2011) Is there a place for cardiovascula magnetic resonance imaging in the evaluation of cardiovascular involvement in rheumatic diseases? Semin Arthritis Rheum 41: 488-496. 
10. Stuckey DJ, Carr CA, Tyler DJ, Clarke K (2008) Cine-MRI versus twodimensional echocardiography to measure in vivo left ventricular function in rat heart. NMR Biomed 21: 765-772.

11. Pennell DJ, Sechtem UP, Higgins CB, Manning WJ, Pohost GM, et al (2004) Clinical indications for cardiovascular magnetic resonance (CMR): Consensus Panel report. Eur Heart J 25: 1940-1965.

12. Kim RJ, Fieno DS, Parrish TB, Harris K, Chen EL, et al. (1999) Relationship of MRI delayed contrast enhancement to irreversible injury, infarct age, and contractile function. Circulation 100: 1992-2002.

13. Kitajima K, Maeda T, Watanabe S, Sugimura K (2011) Recent issues in contrast-induced nephropathy. Int J Urol 18: 686-690.

14. Mavrogeni S, Manoussakis MN, Karagiorga TC, Douskou M, Panagiotakos D, et al. (2009) Detection of coronary artery lesions and myocardial necrosis by magnetic resonance in systemic necrotizing vasculitides. Arthritis Rheum 61: $1121-1129$

15. Mavrogeni S, Papadopoulos G, Karanasios E, Georgakopoulos D Manoussakis MN (2010) Microscopic polyangiitis and Kawasaki disease without overt clinical cardiovascular manifestations and with abnormal cardiovascular magnetic resonance findings. Int J Cardiol 141: e17-19.

16. Allanore Y, Vignaux O, Arnaud L, Puéchal X, Pavy S, et al. (2006) Effects of corticosteroids and immunosuppressors on idiopathic inflammatory myopathy related myocarditis evaluated by magnetic resonance imaging. Ann Rheum Dis 65: $249-252$.

17. Singh JA, Woodard PK, Dávila-Román VG, Waggoner AD, Gutierrez FR, et al. (2005) Cardiac magnetic resonance imaging abnormalities in systemic lupus erythematosus: a preliminary report. Lupus 14: 137-144.

18. Seneviratne MG, Grieve SM, Figtree GA, Garsia R, Celermajer DS, et al (2016) Prevalence, distribution and clinical correlates of myocardial fibrosis in systemic lupus erythematosus: acardiac magnetic resonance study. Lupu 25: $573-581$
19. Giles JT, Malayeri AA, Fernandes V, Post W, Blumenthal RS, et al. (2010) Left ventricular structure and function in patients with rheumatoid arthritis, as assessed by cardiac magnetic resonance imaging. Arthritis Rheum 62 : 940-951.

20. Kobayashi Y, Giles JT, Hirano M, Yokoe I, Nakajima Y, et al. (2010) Assessment of myocardial abnormalities in rheumatoid arthritis using a comprehensive cardiac magnetic resonance approach: a pilot study. Arthritis Res Ther 12: R171.

21. Kobayashi Y, Kobayashi H, Gilea JT, Hirano M, Nakjima Y, et al. (2015) Association of Tocilizumab treatment with Changes in measures of regional left Ventricular function in rheumatoid arthritis, as Assessed by Cardiac Magnetic Resonance Imaging. Int J Rheum Dis.

22. Kobayashi H, Kobayashi $\mathrm{Y}$, Giles JT, Yoneyama K, Nakajima $\mathrm{Y}$, et al. (2014) Tocilizumab treatment increases left ventricular ejection fraction and decreases left ventricular mass index in patients with rheumatoid arthritis without cardiac symptoms: assessed using 3.0 tesla cardiac magnetic resonance imaging. J Rheumatol 41: 1916-1921.

23. Ntusi NA, Piechnik SK, Francis JM, Ferreira VM, Matthews PM, et al. (2015) Diffuse Myocardial Fibrosis and Inflammation in Rheumatoid Arthritis: Insights From CMR T1 Mapping. JACC Cardiovasc Imaging 8: 526-536.

24. Holmström M, Koivuniemi R, Korpi $\mathrm{K}$, Kaasalainen $\mathrm{T}$, Laine $\mathrm{M}$, et al. (2016) Cardiac magnetic resonance imaging reveals frequent myocardial involvement and dysfunction in active rheumatoid arthritis. Clin Exp Rheumatol 34: 416-423.

25. Miszalski-Jamka T, Szczeklik W, Sokolowska B, Karwat K, Belzak K, et al. (2013) Standard and feature tracking magnetic resonance evidence of myocardial involvement in Churg-Strauss syndrome and granulomatosis with polyangiitis (Wegener's) in patients with normal electrocardiograms and transthoracic echocardiography. Int J Cardiovasc Imaging 29: 843-853. 